\title{
Direct Numerical Simulation of Acoustic Noise Generation from the Nozzle Wall of a Hypersonic Wind Tunnel
}

\author{
Junji Huang* and Lian Duan ${ }^{\dagger}$ \\ Missouri University of Science and Technology, Rolla, MO 65409 \\ Meelan M. Choudhari ${ }^{\ddagger}$ \\ NASA Langley Research Center, Hampton, VA 23681
}

\begin{abstract}
The acoustic radiation from the turbulent boundary layer on the nozzle wall of a Mach 6 Ludwieg Tube is simulated using Direct Numerical Simulations (DNS), with the flow conditions falling within the operational range of the Mach 6 Hypersonic Ludwieg Tube, Braunschweig (HLB). The mean and turbulence statistics of the nozzle-wall boundary layer show good agreement with those predicted by Pate's correlation and Reynolds Averaged Navier-Stokes (RANS) computations. The rms pressure fluctuation $p_{r m s}^{\prime} / \tau_{w}$ plateaus in the freestream core of the nozzle. The intensity of the freestream noise within the nozzle is approximately $20 \%$ higher than that radiated from a single flat pate with a similar freestream Mach number, potentially because of the contributions to the acoustic radiation from multiple azimuthal segments of the nozzle wall.
\end{abstract}

\section{Nomenclature}

$c_{f} \quad$ wall skin friction coefficient, dimensionless

$C_{p} \quad$ heat capacity at constant pressure, $\mathrm{J} /(\mathrm{K} \cdot \mathrm{kg})$

$C_{p p}$ two-point correlation coefficient of the pressure signal, dimensionless

$C_{v}$ heat capacity at constant volume, $\mathrm{J} /(\mathrm{K} \cdot \mathrm{kg})$

$H \quad$ shape factor, $H=\delta^{*} / \theta$, dimensionless

$M \quad$ Mach number, dimensionless

$\operatorname{Pr} \quad$ Prandtl number, $\operatorname{Pr}=0.71$, dimensionless

$R \quad$ ideal gas constant, $R=287, \mathrm{~J} /(\mathrm{K} \cdot \mathrm{kg})$, or radius of the axisymmetric nozzle, $\mathrm{m}$

$R e_{\theta}$ Reynolds number based on momentum thickness and freestream viscosity, $R e_{\theta} \equiv \frac{\rho_{\infty} U_{\infty} \theta}{\mu_{\infty}}$, dimensionless

$R e_{\delta_{2}}$ Reynolds number based on momentum thickness and wall viscosity, $R e_{\delta_{2}} \equiv \frac{\rho_{\infty} U_{\infty} \theta}{\mu_{w}}$, dimensionless

$R e_{\tau}$ Reynolds number based on shear velocity and wall viscosity, $R e_{\tau} \equiv \frac{\rho_{w} u_{\tau} \delta}{\mu_{w}}$, dimensionless

rms root mean square

$T$ temperature, $\mathrm{K}$

$T_{r} \quad$ recovery temperature, $T_{r}=T_{\infty}\left(1+0.9 * \frac{\gamma-1}{2} M_{\infty}^{2}\right), \mathrm{K}$

$U_{b} \quad$ bulk convection speed, $\mathrm{m} / \mathrm{s}$

$U_{\infty}$ freestream velocity, $\mathrm{m} / \mathrm{s}$

a speed of sound, $\mathrm{m} / \mathrm{s}$

$p$ pressure, $\mathrm{Pa}$

$q \quad$ dynamic pressure, $\mathrm{Pa}$

$r$ radial coordinate

$u \quad$ streamwise velocity, $\mathrm{m} / \mathrm{s}$

*Graduate Student, Student Member, AIAA

${ }^{\dagger}$ Assistant Professor, Senior Member, AIAA

¥Aerospace Technologist, Computational AeroSciences Branch, M.S. 128. Associate Fellow, AIAA 


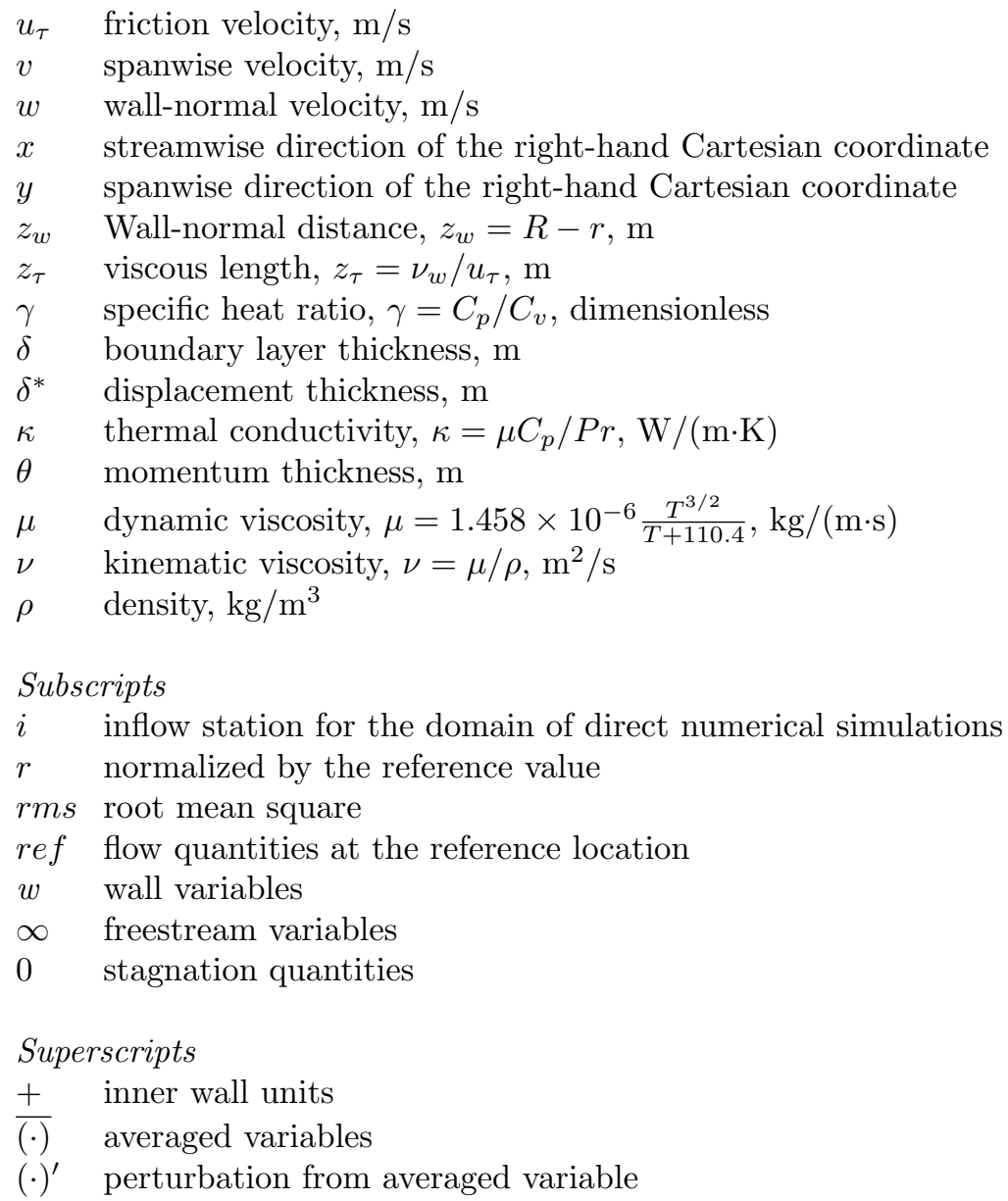

\section{Introduction}

The elevated freestream disturbance levels in conventional (i.e., noisy) high-speed wind tunnels usually result in an earlier onset of transition relative to that in a flight environment or in a quiet tunnel. Yet, the conventional facilities continue to be used for transition sensitive measurements because of the size and Reynolds number limitations of existing quiet facilities and the prohibitive cost of flight tests. To enable a better use of transition data from the conventional facilities, it is important to understand the acoustic fluctuation field that dominates the freestream disturbance environment in those facilities. With increased knowledge of the receptivity mechanisms of high-speed boundary layers, ${ }^{1,2}$ it becomes particularly important to characterize the details of the tunnel acoustics originating from the tunnel-wall turbulent boundary layers.

Direct numerical simulations (DNS) of the acoustic fluctuation field radiated from tunnel-wall turbulent boundary layers can overcome a number of difficulties encountered during experimental measurements of tunnel freestream disturbances and also provide access to quantities that cannot be measured easily. ${ }^{3-5}$ Successful application of DNS for capturing the freestream acoustic pressure fluctuations has been demonstrated for spatially-developing turbulent boundary layers over a flat plate at Mach 2.5, 6, and $14 .{ }^{6-8}$ These flat-plate simulations have the benefits of more easily isolating the acoustic radiation from a single surface, thus facilitating a comprehensive understanding of the freestream disturbance field and its dependence on boundary-layer parameters (e.g., freestream Mach number, wall temperature, Reynolds number). Given that most hypersonic wind tunnels are axisymmetric, DNS of acoustic radiation have also been conducted in a cylindrical domain to study the effect of an axisymmetric enclosure on the noise field. ${ }^{9}$ By choosing a cylindrical geometry, the effects of surface curvature and pressure gradient in the streamwise direction have been neglected, which facilitates a direct comparison with acoustic radiation from a single flat wall in an unconfined setting.

Motivated by the need to enable practical applications of the simulation data for freestream disturbances in the context of actual wind-tunnel experiments and to guide the measurement of tunnel disturbances in 
high-speed facilities, the present study extends the previous simulations of acoustic radiation to those in a realistic wind-tunnel nozzle.

The paper is structured as follows. The flow conditions and numerical methods are outlined in Section II. Section III presents results of a preliminary DNS of the turbulent boundary layer on the inner surface of a hypothetical straight nozzle. A summary of the overall findings is given in Section IV.

\section{Flow Conditions and Numerical Methodology}

Freestream conditions used in the DNS set up are summarized in Table 1, which fall within the range of nozzle exit conditions of Hypersonic Ludwieg Tube, Braunschweig (HLB). ${ }^{10}$ The reference boundary layer thickness, $\delta_{r}$, is chosen to be that at the nozzle exit (or the entrance of test section), where the turbulence statistics are collected and compared with the existing Reynolds-Averaged Navier-Stokes (RANS) (or experimental) data.

Table 1. Nominal freestream conditions for the Mach 6 HLB nozzle exit.

\begin{tabular}{cccccccc}
\hline \hline$M_{\infty}$ & $P_{0}(\mathrm{KPa})$ & $T_{0}(\mathrm{~K})$ & $U_{\infty}(\mathrm{m} / \mathrm{s})$ & $T_{\infty}(\mathrm{K})$ & $\rho_{\infty}\left(\mathrm{kg} / \mathrm{m}^{3}\right)$ & $R e^{-1}(1 / m)$ & $\delta_{r}(\mathrm{~mm})$ \\
\hline 5.7 & 724 & 470 & 903.48 & 62.4 & 0.036 & $7.73 \times 10^{6}$ & 37.0 \\
\hline \hline
\end{tabular}

\section{A. Governing Equations and Numerical Methods}

The full three-dimensional compressible Navier-Stokes equations in conservation form are solved numerically in cylindrical coordinates. The governing flow equations are solved with a collocated grid; hence, a solution point exists at the polar axis $(r=0)$. The singularity at the polar axis manifested in the $1 / r$ terms of the governing equations is handled by using a technique based on power series expansions, ${ }^{11}$ which has been shown to enable the computation of nonaxisymmetric flows in cylindrical coordinates by using highly accurate finite-difference schemes on nonstaggered grids.

The working fluid is assumed to be a perfect gas and the usual constitutive relations for a Newtonian fluid are used: the viscous stress tensor is linearly related to the rate-of-strain tensor, and the heat flux vector is linearly related to the temperature gradient through Fourier's law. The coefficient of viscosity $\mu$ is computed from Sutherland's law, and the coefficient of thermal conductivity $\kappa$ is computed from $\kappa=\mu C_{p} / P r$, with the molecular Prandtl number $P r=0.71$. A detailed description of the governing equations can be found in $\mathrm{Wu}$ et al. ${ }^{12}$

The inviscid fluxes of the governing equations are computed using a seventh-order weighted essentially nonoscillatory (WENO) scheme. Compared with the original finite-difference WENO introduced by Jiang and $\mathrm{Shu},{ }^{13}$ the present scheme is optimized by means of limiters ${ }^{12,14}$ to reduce the numerical dissipation. The viscous fluxes are discretized using a fourth-order central difference scheme and time integration is performed using a third-order low-storage Runge-Kutta scheme. ${ }^{15}$

The details of the DNS methodology have been documented in our previous simulations of acoustic radiation from turbulent boundary layers. ${ }^{6,7,9}$ The DNS solver has been previously shown to be suitable for computing transitional and fully turbulent flows, including hypersonic turbulent boundary layers, ${ }^{16,17}$ the propagation of linear instability waves in 2D high-speed boundary layers, and secondary instability and laminar breakdown of swept-wing boundary layers. ${ }^{18,19}$

\section{B. Simulation Setup and Boundary Conditions}

Numerical calculations of boundary layer flow within the valve-controlled hypersonic Ludwieg tube have been carried out by the group of Prof. Rolf Radespiel in Technische Universität Braunschweig (TUB), Germany, using the TAU-Code. ${ }^{10,20-22}$ Their RANS calculations simulate the full-domain HLB geometry, including the storage tube, the fast acting valve, the Laval nozzle and the test section. The flow is assumed to be fully turbulent throughout the nozzle; and the Reynolds stresses and turbulent heat flux are modeled via Menter's two equation, shear stress transport (SST) turbulence model. As seen in the top of Figure 1, the HLB configuration starts from the storage tube ahead of the nozzle throat, which is located at $x \approx 1.41 \mathrm{~m}$. 
The nozzle part spans from $x \approx 1.41 \mathrm{~m}$ to $x \approx 3.8 \mathrm{~m}$, followed by the test section region. The RANS simulation will be referred as Case "RANS_HLB" in the remainder of the paper.

The expansion portion of the Laval nozzle is simulated using DNS. The DNS domain starts slightly downstream of the nozzle throat at $x=2.0 \mathrm{~m}$ with a local freestream Mach number of $M_{\infty}=3.84$ and ends at the nozzle exit at $x=3.8 \mathrm{~m}$ with a freestream Mach number of $M_{\infty}=5.71$. The selected DNS domain covers the origin of most of the acoustic sources responsible for generating freestream noise in the test section as it includes the portion of the nozzle with high freestream Mach numbers, and thus large intensity of noise radiation. The streamwise length of the DNS domain is $48.67 \delta_{r}$, where $\delta_{r}=0.037 \mathrm{~m}$ is the boundary-layer thickness at the nozzle exit predicted by Case RANS_HLB. The streamwise domain length is long enough for the artificial inflow turbulence to develop based on our previous experience of DNS over a zero-pressure-gradient flat plate. ${ }^{23}$ To prevent the reflection of any noise generated by the numerical treatment of outflow boundary condition, an extra region $\left(11.4 \delta_{r}\right)$ is appended to the DNS domain of interest $(x<3.8 \mathrm{~m})$, wherein the grid is progressively stretched in the streamwise direction. The grid dimensions and resolution of DNS are shown in Table 3 . The cylindrical grid for the DNS domain comprises $2500 \times 2000 \times 500$ points in streamwise, azimuthal and radial directions, respectively. The streamwise grid distribution in Case RANS_HLB is interpolated and used in the streamwise direction of DNS. The maximum grid spacing in the streamwise direction within the domain of interest is $\Delta x^{+}=8.8$, where the viscous length scale $z_{\tau}$ is based on the RANS solution for Case RANS_HLB at the nozzle exit location, $x=3.8 \mathrm{~m}$. A uniform grid distribution is used in the azimuthal direction so that the highest linear grid spacing is at the wall region of the nozzle exit, $\Delta y^{+}=8.2$. Grid distribution in the radial direction is clustered near the nozzle wall with the near-wall grid spacing at the nozzle exit equal to $\Delta r^{+}=0.63$, and is kept uniform $\left(\Delta r^{+}=6.5\right)$ in the free stream all the way to the nozzle axis. The above radial grid distribution ensures that both boundary layer and freestream regions are well resolved in the current DNS.

For the inlet boundary condition, the non-uniform digital-filtering (DF) method ${ }^{23,24}$ is applied to generate inflow turbulence for the nozzle flow. The mean boundary layer profiles and the Reynolds stress tensor at the inflow are extracted from Case RANS_HLB at $x=2.0 \mathrm{~m}$. The method is modified by using a change-ofbasis transformation for the second-order tensors on the curvilinear, non-uniform grid. The robustness and adequacy of the DF inflow method for predicting the global pressure field induced by hypersonic turbulent boundary layers has been demonstrated on both flat plate $^{23}$ and axisymmetric nozzle ${ }^{9}$ configurations. Consistent with previous DNS of acoustic radiation from a flat plate using the turbulence recycling technique, the artificial inflow turbulence is only imposed within a short distance $\left(3.5 \delta_{i}\right)$ from the nozzle wall. On the nozzle wall, no-slip conditions are applied for the three velocity components, and an isothermal condition is used for the temperature with $T_{w} \approx 1.0 T_{r}$. The surface temperature was close to adiabatic in order to match the the wall temperature distribution of the RANS solution provided by TUB (Case RANS_HLB). In a follow on simulation, the effect of surface temperature on the acoustic radiation characteristics will be investigated by considering a wall temperature distribution that is close to the room temperature value in the vicinity of the test section. At the outflow boundary, unsteady nonreflecting boundary conditions based on Thompson ${ }^{25}$ are imposed after introducing an outflow zone with a gradually stretched axial grid beyond the nozzle exit location. The polar-axis boundary condition mentioned above is used at the nozzle axis to remove the coordinate singularity in the axisymmetric grid. Finally, periodic boundary conditions are used in the azimuthal direction. It should be noted that although the reference location is at the nozzle exit $(x=3.8 \mathrm{~m})$, the velocity and pressure statistics at $x=3.7 \mathrm{~m}$ are analyzed instead as the axial grid stretching starts at $x=3.8 \mathrm{~m}$ in the present DNS; and the change in grid resolution is found to influence the local fluctuating signals. Future simulations will remedy this flaw by including part of the tunnel test section into the computational domain and by delaying the onset of grid stretching at an axial location farther downstream.

Table 2. Boundary layer properties at nozzle exit $x=3.8 \mathrm{~m}$.

\begin{tabular}{lcrrrrccccc}
\hline \hline Case & $T_{w}(\mathrm{~K})$ & \multicolumn{1}{c}{$T_{w} / T_{r}$} & \multicolumn{1}{c}{$R e_{\theta}$} & \multicolumn{1}{c}{$R e_{\tau}$} & $R e_{\delta 2}$ & $\delta(\mathrm{mm})$ & $\delta^{*}(\mathrm{~mm})$ & $\mathrm{H}$ & $z_{\tau}(\mu \mathrm{m})$ & $c_{f}\left(10^{-3}\right)$ \\
\hline Pate & 419.0 & 0.98 & 10480.3 & 452.9 & 1975.8 & 38.3 & 21.5 & 14.1 & 84.5 & 1.12 \\
RANS_HLB & 419.0 & 0.98 & 10485.5 & 386.8 & 1846.0 & 37.0 & 20.8 & 15.3 & 95.7 & 0.74 \\
DNS_HLB & 419.0 & 0.98 & 9858.4 & 380.1 & 1708.2 & 37.5 & 18.2 & 14.6 & 98.6 & 0.70 \\
\hline \hline
\end{tabular}


Table 3. Domain and mesh parameters for Cases DNS_HLB.

\begin{tabular}{lccccccccc}
\hline \hline Case & $N_{x}$ & $N_{y}$ & $N_{z}$ & $L_{x} / \delta_{r}$ & $R_{\text {exit }} / \delta_{r}$ & $\Delta x_{\max }^{+}$ & $\Delta y_{\max }^{+}$ & $\Delta z_{\text {wall }}^{+}$ & $\Delta z_{\text {inf }}^{+}$ \\
\hline DNS_HLB & 2500 & 2000 & 500 & 60.1 & 6.8 & 8.8 & 8.2 & 0.63 & 6.5 \\
\hline \hline
\end{tabular}

\section{Results}

In this section, DNS results for the nozzle-wall turbulent boundary layer are presented. The mean turbulent statistics within the boundary layer are compared with Case RANS_HLB, and the pressure fluctuations radiated from the boundary layer toward the interior of the nozzle are also analyzed. Results of a DNS of hypersonic turbulent boundary layer over a flat plate with $M_{\infty}=5.86$ and $T_{w} / T_{r}=0.76^{7}$ (Case DNS_FlatPlate) are also plotted for comparison.

Table 2 lists the properties of the turbulent boundary layer at $x=3.8 \mathrm{~m}$. Three sets of results are included in the Table, based on the RANS calculations (Case RANS_HLB), the DNS (Case DNS_HLB), and the empirical estimates derived from the Pate correlation. ${ }^{26}$ The latter correlation allows one to deduce the displacement boundary layer thickness, $\delta^{*}$, and the nozzle wall friction coefficient, $c_{f}$. The shape factor $H$ of the tunnel-wall boundary layer is then estimated using an empirical relation by Wood: ${ }^{27}$

$$
H=\left(1+\frac{\gamma-1}{2} M_{\infty}^{2}\right) \frac{T_{w}}{T_{0}} H_{i}+\frac{0.9}{2}(\gamma-1) M_{\infty}^{2}
$$

where the incompressible shape factor $H_{i}$ is given by $H_{i}=(n+2) / n=11 / 9$, assuming a $1 / 9$ power law profile $(n=9)$. Given the value of $H$ and $\delta^{*}$, the momentum thickness can be calculated as

$$
\theta=\delta^{*} / H
$$

The DNS-predicted integral parameters of the nozzle-wall boundary layer compare well with those predicted by the empirical corelation of Pate. ${ }^{26}$ Good comparison is also achieved for the freestream Mach number distribution along the nozzle axis among DNS, RANS, and the theory (Figure 2a). Figure 2b shows the wall-normal distribution of the normalized streamwise velocity $x=3.7$. Some noticeable differences exist in streamwise velocity profile at the outer portion of the boundary layer between the DNS and the RANS. Figures 3a to 3d show the normalized Reynolds stress distributions at $x=3.7 \mathrm{~m}$. For Case RANS_HLB, the Reynolds stress components are calculated by using the Boussinesq approximation. The streamwise normal Reynolds stress is significantly underpredicted by the RANS. However, the predictions for the Reynolds shear stress match rather well between the DNS and RANS results.

Figure 4a plots the streamwise distribution of rms pressure fluctuations at the wall and also in the free stream $\left(z_{w} / \delta_{r}=2.5\right)$, i.e., core of the nozzle. Both fluctuation signals become approximately homogeneous in the streamwise direction for $x>2.5 \mathrm{~m}$. Specifically, the variation of rms pressure fluctuation along the wall-normal direction is shown in Figure 4b. The result of DNS data for the Mach 6 turbulent boundary layer over a flat plate ${ }^{7}$ is also plotted in the same figure. The wall pressure fluctuations in both cases are very similar to each other. Furthermore, both curves display a small decrease in $p_{r m s}^{\prime} / \tau_{w}$ away from the wall and an interior peak between 20 and 30 wall units. The differences arise in the outer part of the boundary layer, where the pressure fluctuation level in the nozzle case exhibits a faster decay to its freestream (i.e., core flow) level. Further analysis is required to confirm this significant difference and to pinpoint the underlying cause.

The bulk propagation speed $\left(U_{b}\right)$, defined as the value that minimizes the difference between the real time evolution of $p(x, t)$ and a frozen wave approximation $p\left(x-U_{b} t\right),{ }^{6,7}$ is plotted as a function of the wall-normal coordinate in Figure 5. The following expression is used to calculate $U_{b}$ :

$$
U_{b}=-\frac{\overline{(\partial p / \partial t)(\partial p / \partial x)}}{\overline{\partial p / \partial x}}
$$

The bulk propagation speed of the pressure structures is similar to that of the flat plate case, although has a smaller value within the boundary layer. They both approaches a value of approximately $0.7 U_{\infty}$ in the free stream. 
Figures 6,7 , and 8 show the contours of two-point correlations at multiple wall-normal locations both within and outside the boundary layer. Results from Case DNS_FlatPlate are also plotted together for comparison. The good agreement between the FlatPlate and HLB cases can be seen at all heights, indicating that the spatial structure of the boundary-layer induced pressure fluctuation is relatively insensitive to the transverse curvature of the surface. The contours of streamwise wall-normal correlations indicate the preferred orientations for the two cases. For Case DNS_HLB, the angle is approximately $31^{\circ}$, and for Case DNS_FlatPlate, the angle is $26^{\circ}$. Note that the nozzle wall at $x=3.7 \mathrm{~m}$ also forms an angle of $3^{\circ}$ with respect to the axis.

Numerical schlieren images of the radiated acoustic field are shown in Figure 9. The wave fronts exhibit a preferred orientation of $\theta \approx 31^{\circ}$ with respect to the nozzle centerline within the streamwise-radial plane (Figure 9c), a little larger than that seen for the flat plate turbulent boundary layer (Figure 9d), ${ }^{6-8,28}$ where $\theta \approx 26^{\circ}$. The acoustic field within the core of the nozzle clearly shows the simultaneous presence of waves propagating in both upward and downward directions within the streamwise-radial plane. The density gradients in Figure 9b reveal the omnidirectional origin of the acoustic field within a given cross-section of the nozzle, which adds to the stochastic nature of the wave front pattern at a given axial location. Because of the limited length of the nozzle and relatively shallow acoustic propagation angles with respect to the flow direction, the number of acoustic reflections from the nozzle wall that contribute to the acoustic signal at the nozzle exit plane is expected to be small, but further analysis is required to establish the relative contribution from those acoustic reflections, i.e., the reverberation effect.

\section{Summary}

This paper has outlined preliminary findings from ongoing direct numerical simulations of the turbulent boundary layer inside a Mach 6 Ludwieg tube. Specifically, the previous simulations targeting the numerical synthesis of acoustic disturbance environment emanating from a hypersonic boundary layer flow have been extended to a full scale nozzle from an actual wind tunnel facility for the first time. The freestream conditions of the DNS fall within the operating conditions of the HLB at TU Braunschweig. The mean and turbulence statistics of the nozzle-wall turbulent boundary layer predicted by DNS compare well with those of Pate's correlation and the larger domain RANS simulation. The rms pressure fluctuation $p_{r m s}^{\prime} / \tau_{w}$ plateaus in the free stream and is approximately $20 \%$ higher than that induced by the turbulent boundary layer over a single flat plate at a similar freestream Mach number. This increase in the noise intensity is believed to be caused by the combined effect of acoustic radiation arriving from different azimuthal segments of the axisymmetric nozzle wall. The current simulations have highlighted the need for experimental measurements of nozzle boundary layer profiles and also paved the way for improved simulations including the test section downstream of the nozzle exit.

\section{Acknowledgments}

The authors would like to thank Prof. Rolf Radespiel and his group from Technische Universität Braunschweig, Germany, for providing the nozzle geometry of HLB and for conducting RANS calculations of the full wind tunnel. The work is supported by the Air Force Office of Scientific Research under award number FA9550-14-1-0170, managed by Dr. Ivett Leyva. Computational resources are provided by the DoD High Performance Computing Modernization Program. Any opinions, findings, and conclusions or recommendations expressed in this material are those of the authors and do not necessarily reflect the views of the United States Air Force.

\section{References}

\footnotetext{
${ }^{1}$ Fedorov, A. V., "Receptivity of a High-Speed Boundary Layer to Acoustic Disturbances," Journal of Fluid Mechanics, Vol. 491, 2003, pp. 101-129.

${ }^{2}$ Zhong, X. and Wang, X., "Direct Numerical Simulation on the Receptivity, Instability, and Transition of Hypersonic Boundary Layers," Annu. Rev. Fluid Mech., Vol. 44, 2012, pp. 527-561.

${ }^{3}$ Laufer, J., "Some Statistical Properties of the Pressure Field Radiated by a Turbulent Boundary Layer," Physics of Fluids, Vol. 7, No. 8, 1964, pp. 1191-1197.

${ }^{4}$ Donaldson, J. and Coulter, S., "A Review of Free-Stream Flow Fluctuation and Steady-State Flow Quality Measurements in the AEDC/VKF Supersonic Tunnel A and Hypersonic Tunnel B," AIAA Paper 95-6137, 1995.
} 
${ }^{5}$ Schneider, S. P., "Effects of High-Speed Tunnel Noise on Laminar-Turbulent Transition," Journal of Spacecraft and Rockets, Vol. 38, No. 3, 2001, pp. 323-333.

${ }^{6}$ Duan, L., Choudhari, M. M., and Wu, M., "Numerical Study of Pressure Fluctuations due to a Supersonic Turbulent Boundary Layer," Journal of Fluid Mechanics, Vol. 746, 2014, pp. 165-192.

${ }^{7}$ Duan, L., Choudhari, M. M., and Zhang, C., "Pressure fluctuations induced by a hypersonic turbulent boundary layer," Journal of Fluid Mechanics, Vol. 804, 2016, pp. 578-607.

${ }^{8}$ Duan, L. and Choudhari, M. M., "Analysis of Numerical Simulation Database for Pressure Fluctuations Induced by High-Speed Turbulent Boundary Layers," AIAA Paper 2014-2912, 2014.

${ }^{9}$ Huang, J., Zhang, C., Duan, L., and Choudhari, M. M., "Direct Numerical Simulation of Hypersonic Turbulent Boundary Layers inside an Axisymmetric Nozzle," 55th AIAA Aerospace Sciences Meeting, 2017, p. 0067.

${ }^{10}$ Radespiel, R., Estorf, M., Heitmann, D., Muñoz, F., and Wolf, T., "Hypersonic Ludwieg Tube," Experimental Methods of Shock Wave Research, Springer, 2016, pp. 433-458.

${ }^{11}$ Constantinescu, G. S. and Lele, S. K., "A Highly Accurate Technique for the Treatment of Flow Equations at the Polar Axis in Cylindrical Coordinates Using Series Expansions," Journal of Computational Physics, Vol. 183, 2002, pp. 165-186.

${ }^{12} \mathrm{Wu}, \mathrm{M}$. and Martín, M. P., "Direct numerical simulation of supersonic boundary layer over a compression ramp," AIAA Journal, Vol. 45, No. 4, 2007, pp. 879-889.

${ }^{13}$ Jiang, G. S. and Shu, C. W., "Efficient Implementation of Weighted ENO Schemes," Journal of Computational Physics, Vol. 126, No. 1, 1996, pp. 202-228.

${ }^{14}$ Taylor, E. M., Wu, M., and Martín, M. P., "Optimization of Nonlinear Error Sources for Weighted Non-Oscillatory Methods in Direct Numerical Simulations of Compressible Turbulence," Journal of Computational Physics, Vol. 223, No. 1, 2006 , pp. 384-397.

${ }^{15}$ Williamson, J., "Low-Storage Runge-Kutta Schemes," Journal of Computational Physics, Vol. 35, No. 1, 1980 , pp. 48-56.

${ }^{16}$ Martín, M., "DNS of Hypersonic Turbulent Boundary Layers. Part I: Initialization and Comparison with Experiments," Journal of Fluid Mechanics, Vol. 570, 2007, pp. 347-364.

${ }^{17}$ Duan, L., Beekman, I., and Martín, M. P., "Direct Numerical Simulation of Hypersonic Turbulent Boundary Layers. Part 3: Effect of Mach Number," Journal of Fluid Mechanics, Vol. 672, 2011, pp. 245-267.

${ }^{18}$ Duan, L., Choudhari, M. M., Li, F., and Wu, M., "Direct Numerical Simulation of Transition in a Swept-Wing Boundary Layer," AIAA Paper 2013-2617, 2013.

${ }^{19}$ Choudhari, M. M., Li, F., Duan, L., Chang, C.-L., Carpenter, M. H., Streett, C. L., and Malik, M. R., "Towards Bridging the Gaps in Holistic Transition Prediction via Numerical Simulations (Invited)," AIAA Paper 2013-2718, 2013.

${ }^{20}$ Estorf, M., Wolf, T., and Radespiel, R., "Experimental and numerical investigations on the operation of the Hypersonic Ludwieg Tube Braunschweig," Fifth European Symposium on Aerothermodynamics for Space Vehicles, Vol. 563, 2005, p. 579.

${ }^{21}$ Wolf, T., Estorf, M., and Radespiel, R., "Investigation of the starting process in a Ludwieg tube," Theoretical and Computational Fluid Dynamics, Vol. 21, No. 2, 2007, pp. 81-98.

${ }^{22}$ Wolf, T., Estorf, M., and Radespiel, R., "Numerical rebuilding of the flow in a valve-controlled Ludwieg tube," Shock Waves, 2009, pp. 665-670.

${ }^{23}$ Huang, J., Zhang, C., and Duan, L., "Turbulent Inflow Generation for Direct Simulations of Hypersonic Turbulent Boundary Layers and their Freestream Acoustic Radiation," 46th AIAA Fluid Dynamics Conference, 2016, p. 3639.

${ }^{24}$ Dhamankar, N. S., Martha, C. S., Situ, Y., Aikens, K. M., Blaisdell, G. A., and Lyrintzis, A. S., "Digital Filter-Based Turbulent Inflow Generation for Jet Aeroacoustics on Non-Uniform Structured Grids," AIAA Paper 2014-1401, 2014.

${ }^{25}$ Thompson, K. W., "Time Dependent Boundary Conditions for Hyperbolic Systems," Journal of Computational Physics, Vol. 68, No. 1, Jan. 1987, pp. 1-24.

${ }^{26}$ Pate, S. R., "Dominance of radiated aerodynamic noise on boundary-layer transition in supersonic-hypersonic wind tunnels," Tech. rep., Tech. Rep. AEDC-TR-77-107, Arnold Engineering Development Center, 1978.

${ }^{27}$ Wood, N., "Calculation of the turbulent boundary layer in the nozzle of an intermittent axisymmetric hypersonic wind tunnel," 1963.

${ }^{28}$ Duan, L. and Choudhari, M. M., "Numerical Study of Pressure Fluctuations due to a Mach 6 Turbulent Boundary Layer," AIAA Paper 2013-0532, 2013. 


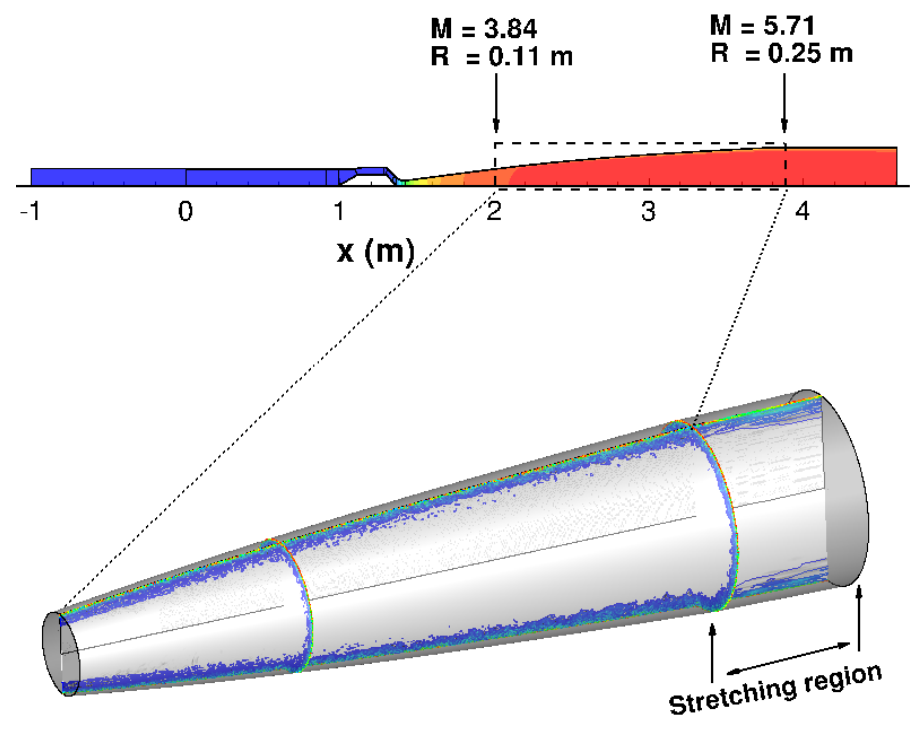

Figure 1. Computational domain set up for (top) Case RANS_HLB, contours colored by Mach number, and (bottom) Case DNS_HLB, shown as numerical schlieren image with contours colored by the magnitude of vorticity to emphasize the large-scale motions within the boundary layer.

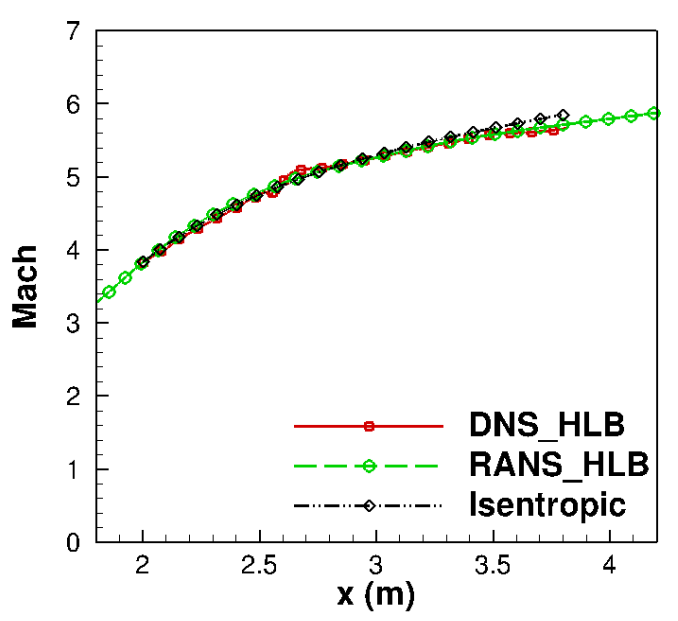

(a)

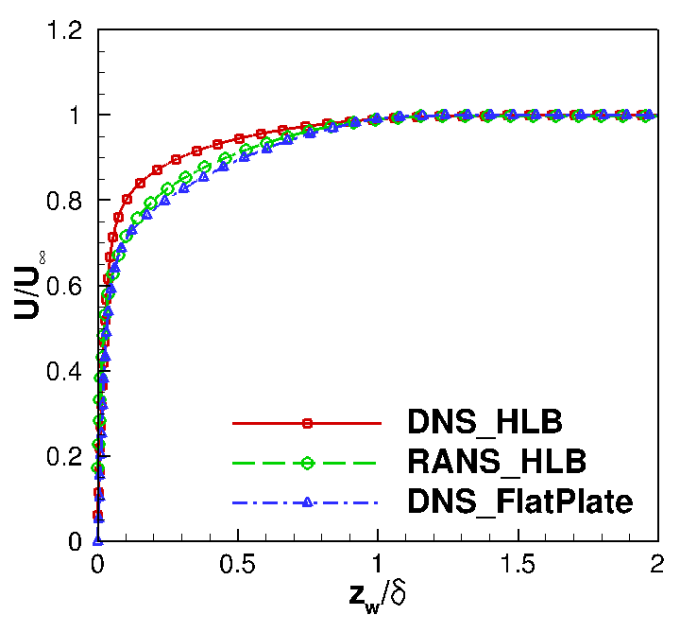

(b)

Figure 2. Comparison between Case DNS_HLB and Case RANS_HLB. (a) Mach distribution along the nozzle axis; (b) normalized streamwise velocity profile at $x=3.7 \mathrm{~m}$. Case DNS_FlatPlate corresponds to the DNS of hypersonic turbulent boundary layer over a flat plate with $M_{\infty}=5.86$ and $T_{w} / T_{r}=0.76 .^{7}$ 


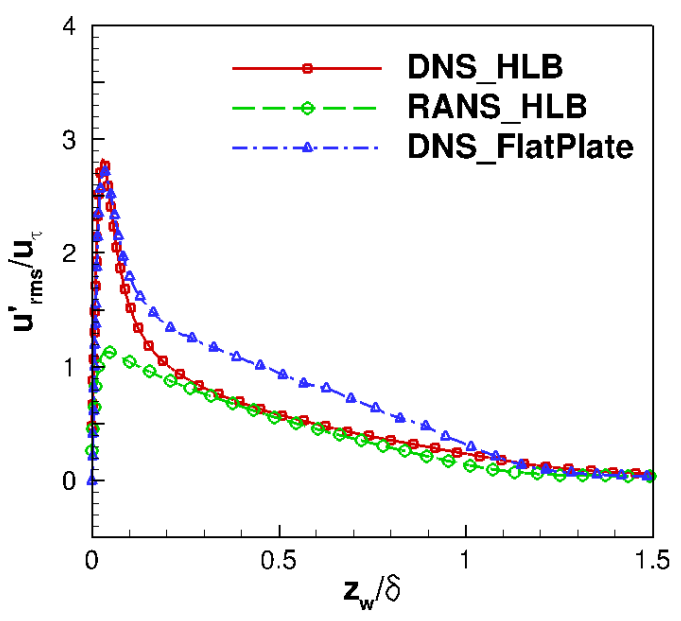

(a)

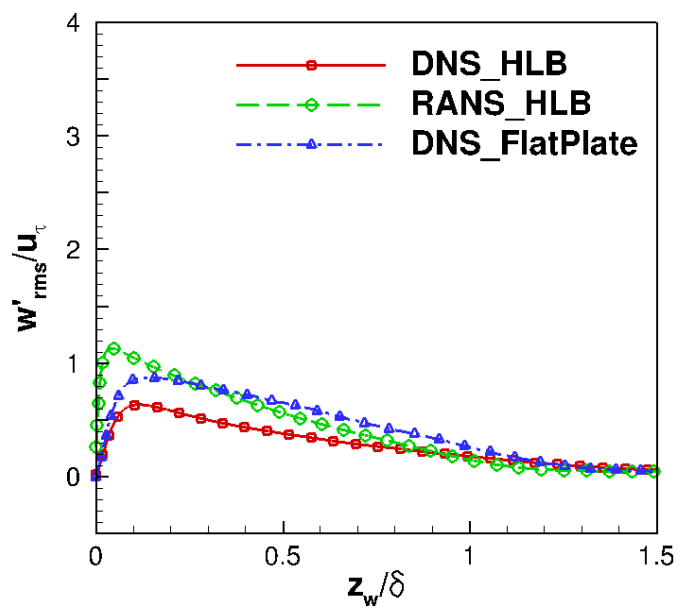

(c)

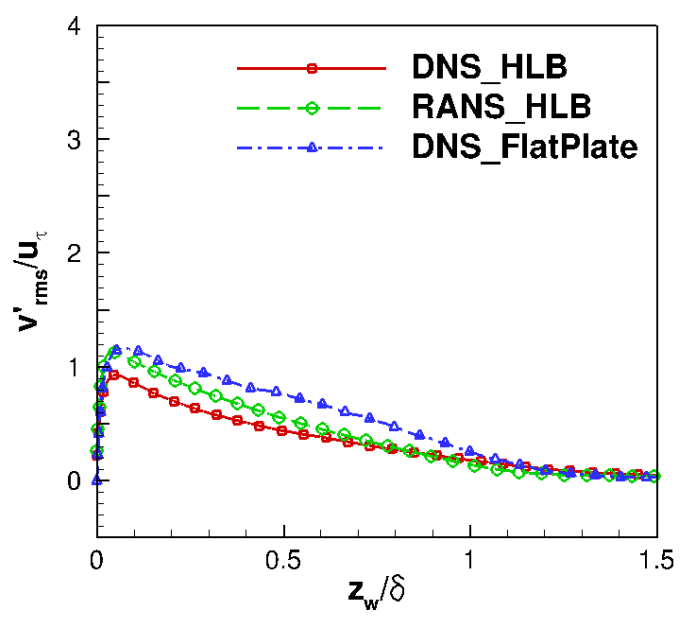

(b)

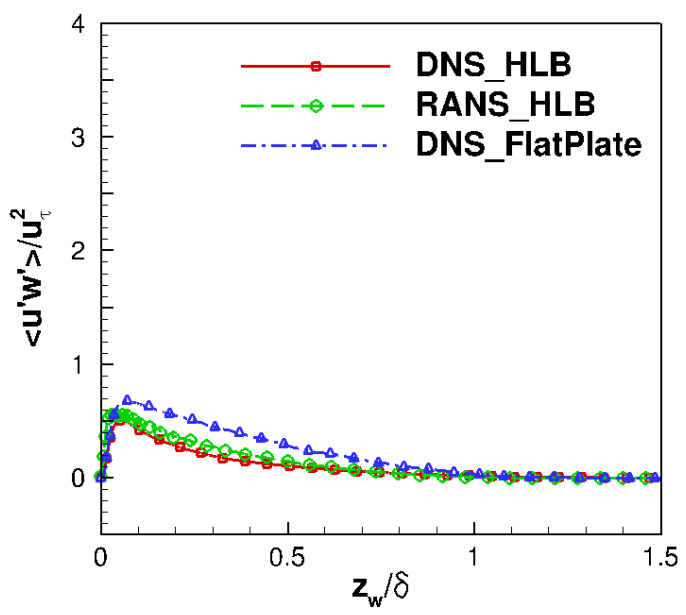

(d)

Figure 3. Comparison of Reynolds stresses at $x=3.7 \mathbf{m}$ between Case DNS_HLB and Case RANS_HLB. (a) $u_{r m s}^{\prime} / u_{\tau}$; (b) $v_{r m s}^{\prime} / u_{\tau}$; (c) $w_{r m s}^{\prime} / u_{\tau}$; (d) $<u^{\prime} w^{\prime}>/ u_{\tau}^{2}$. Case DNS_FlatPlate corresponds to the DNS of hypersonic turbulent boundary layer over a flat plate with $M_{\infty}=5.86$ and $T_{w} / T_{r}=0.76 .^{7}$ 


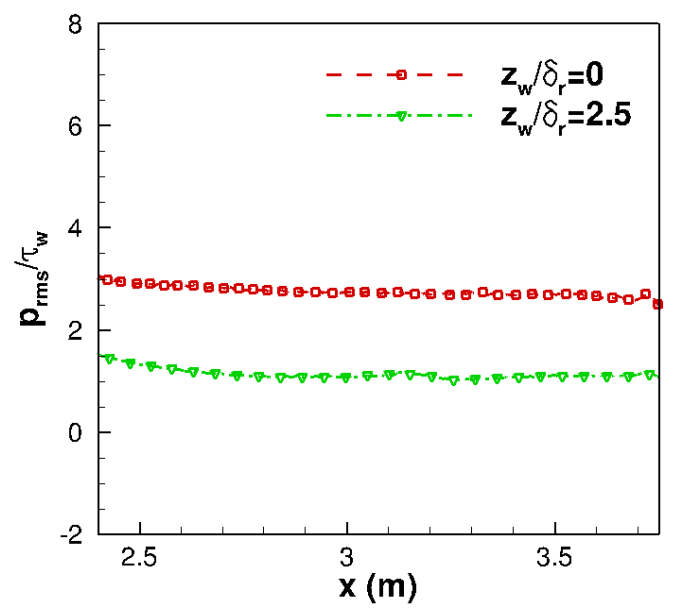

(a)

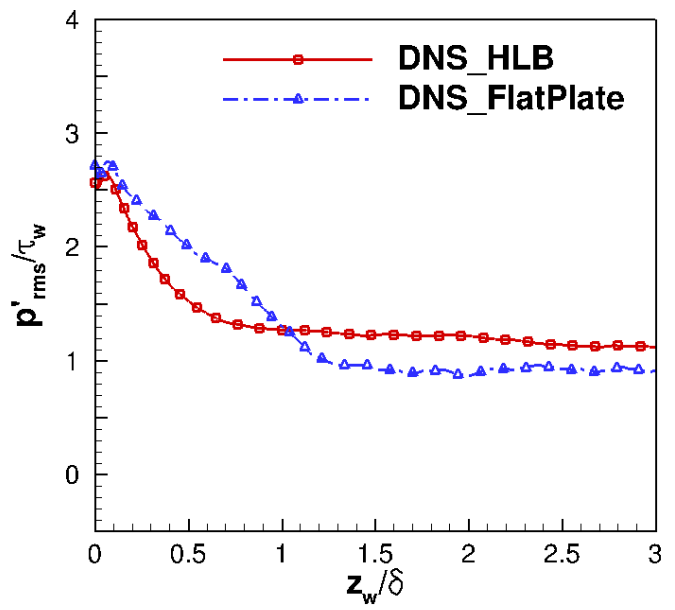

(b)

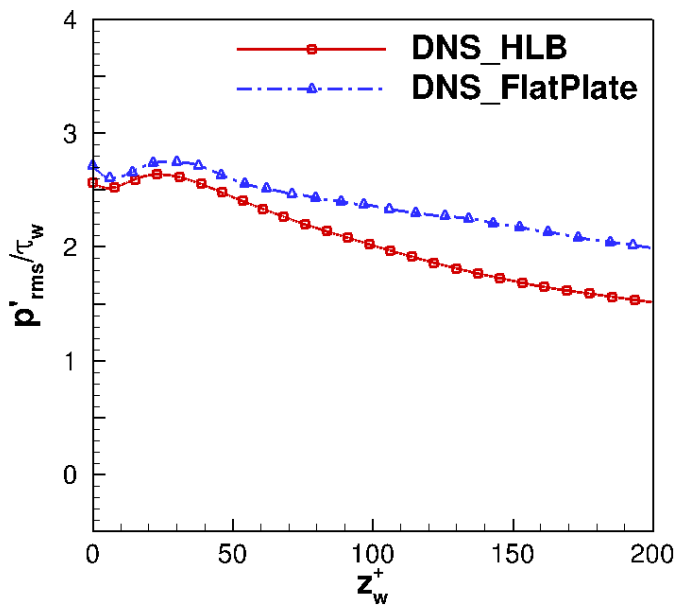

(c)

Figure 4. (a) Evolution of both the wall and freestream normalized rms pressure fluctuation with streamwise distance; rms pressure fluctuation profile at $x=3.7 \mathrm{~m}$ in (b) outer unit, and (c) inner unit. Case DNS_FlatPlate corresponds to the DNS of hypersonic turbulent boundary layer over a flat plate with $M_{\infty}=5.86$ and $T_{w} / T_{r}=0.76 .^{7}$ 


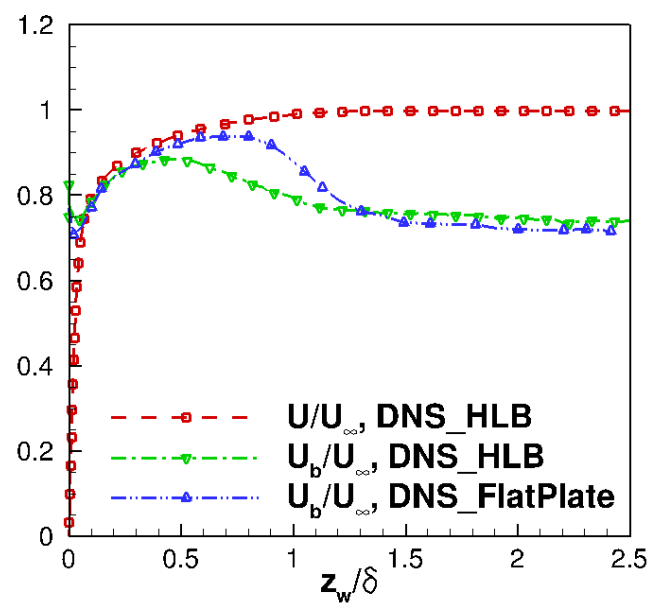

Figure 5. Bulk propagation speed of pressure fluctuations as a function of wall-normal distance at $x=3.7 \mathrm{~m}, R e_{\tau} \approx 390$. Case DNS_FlatPlate corresponds to the DNS of hypersonic turbulent boundary layer over a flat plate with $M_{\infty}=5.86$ and $T_{w} / T_{r}=0.76 .^{7}$ 


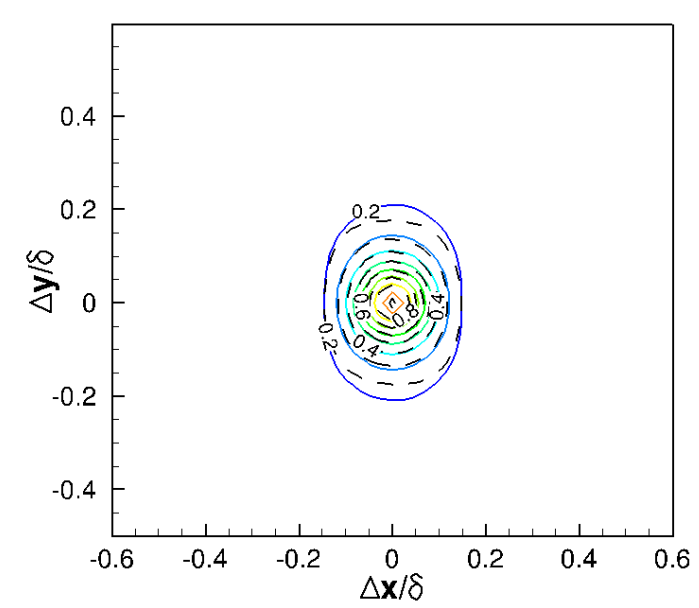

(a) Wall

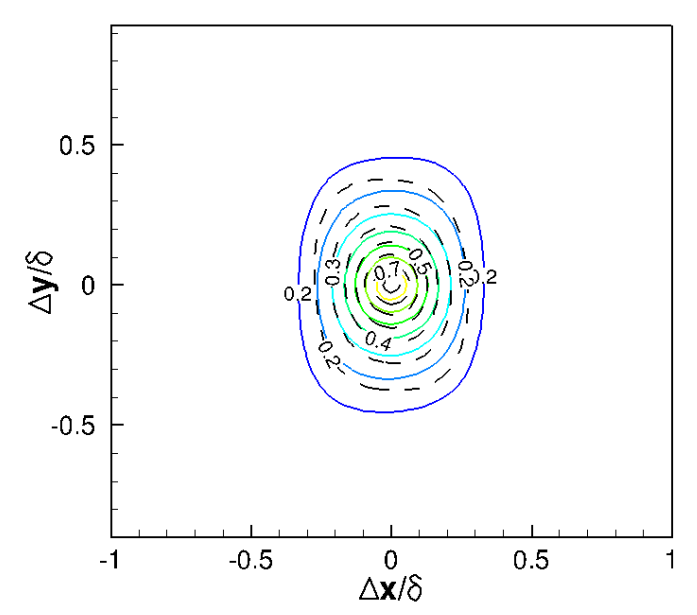

(c) $z_{w, r e f} / \delta=0.83$

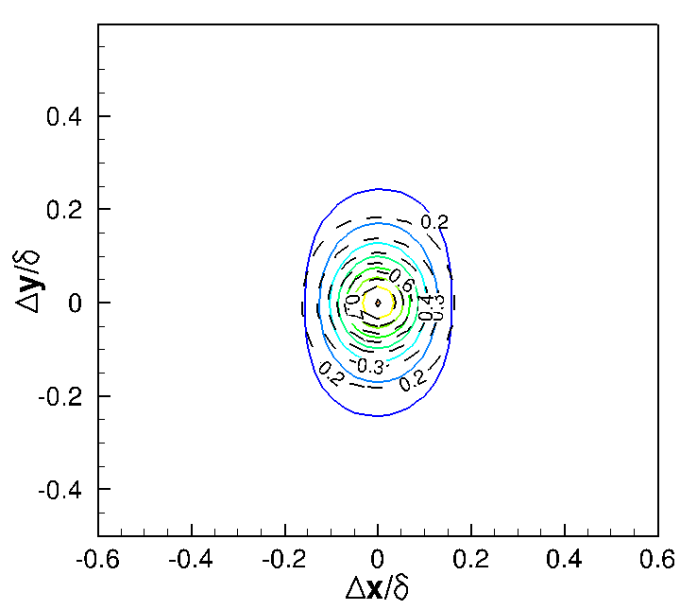

(b) $z_{w, r e f} / \delta=0.18$

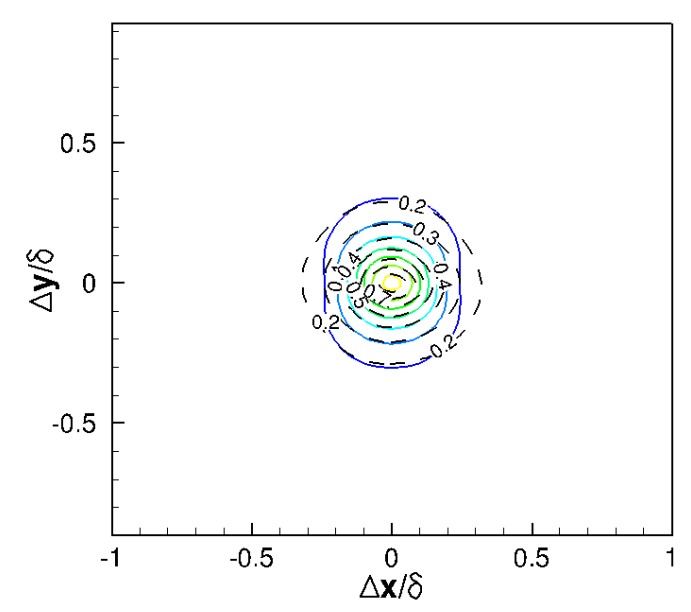

(d) $z_{w, r e f} / \delta=2.97$

Figure 6. Contours of constant streamwise azimuthal (spanwise) correlation coefficient of the pressure signal $C_{p p}(\Delta x, \Delta y)$ at selected heights for Case DNS_HLB (Colored solid line) and Case DNS_FlatPlate (Black dashed line). Case DNS_FlatPlate corresponds to the DNS of hypersonic turbulent boundary layer over a flat plate with $M_{\infty}=5.86$ and $T_{w} / T_{r}=0.76{ }^{7}$ Contours levels vary from 0.2 to 0.9 with increments of 0.1 . 


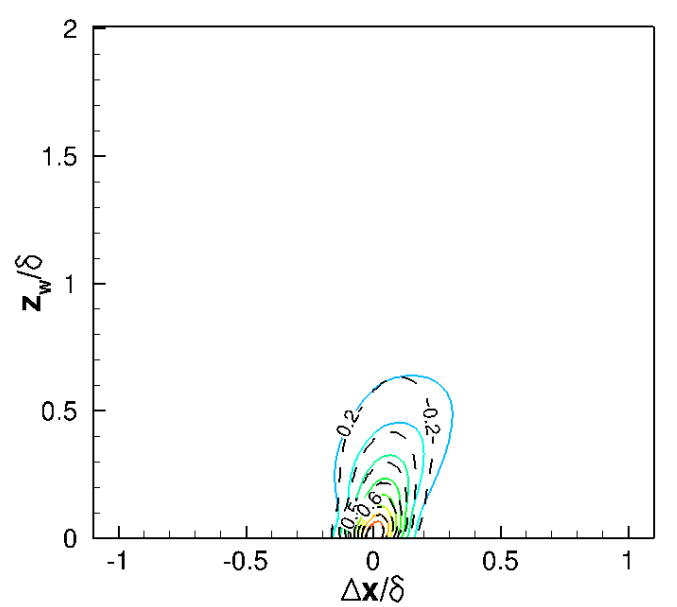

(a) Wall

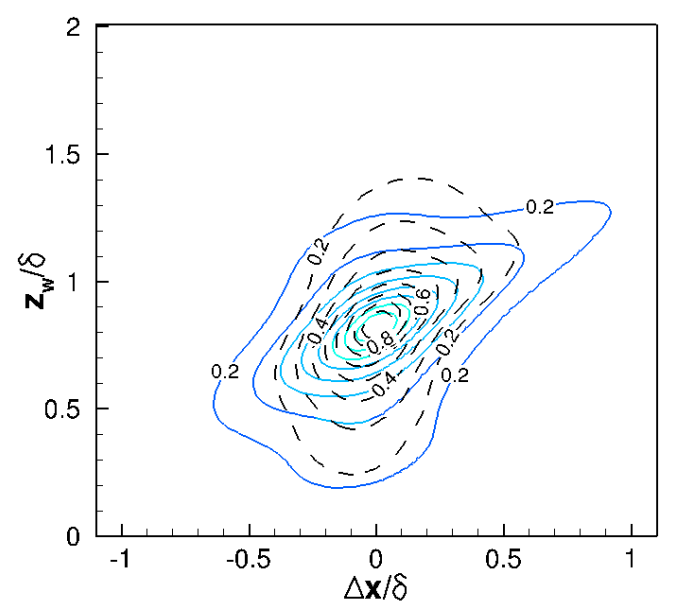

(c) $z_{w, r e f} / \delta=0.83$

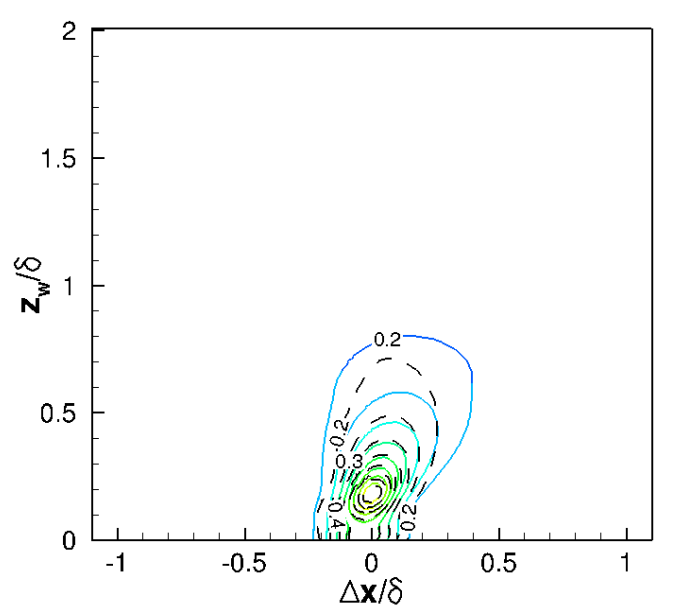

(b) $z_{w, r e f} / \delta=0.18$

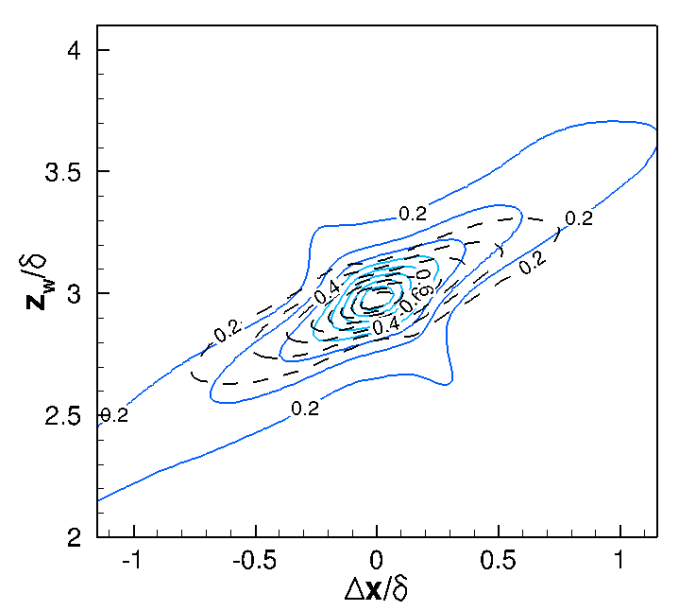

(d) $z_{w, r e f} / \delta=2.97$

Figure 7. Contours of constant streamwise radial (wall-normal) correlation coefficient of the pressure signal $C_{p p}(\Delta x, \Delta y)$ at selected heights for Case DNS_HLB (Colored solid line) and Case DNS_FlatPlate (Black dashed line). Case DNS_FlatPlate corresponds to the DNS of hypersonic turbulent boundary layer over a flat plate with $M_{\infty}=5.86$ and $T_{w} / T_{r}=0.76 .^{7}$ Contours levels vary from 0.2 to 0.9 with increments of 0.1 . 


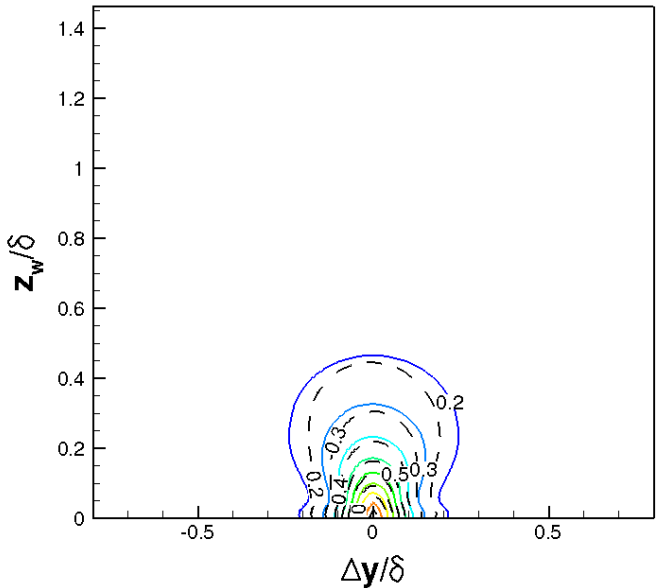

(a) Wall

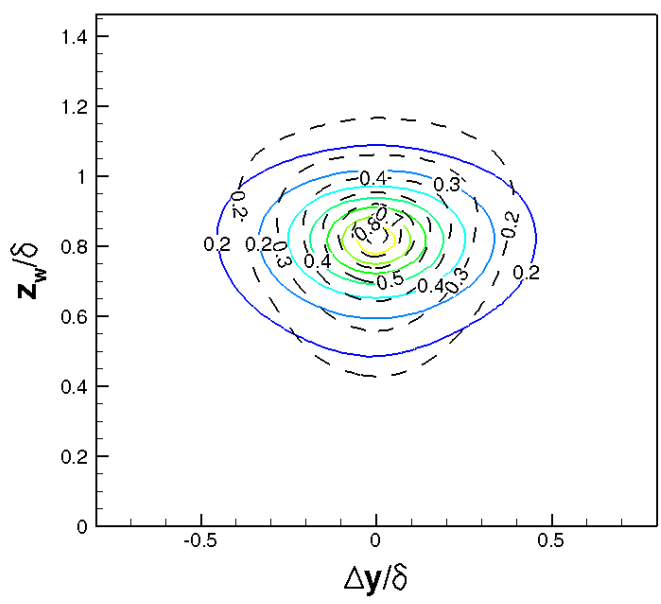

(c) $z_{w, r e f} / \delta=0.83$

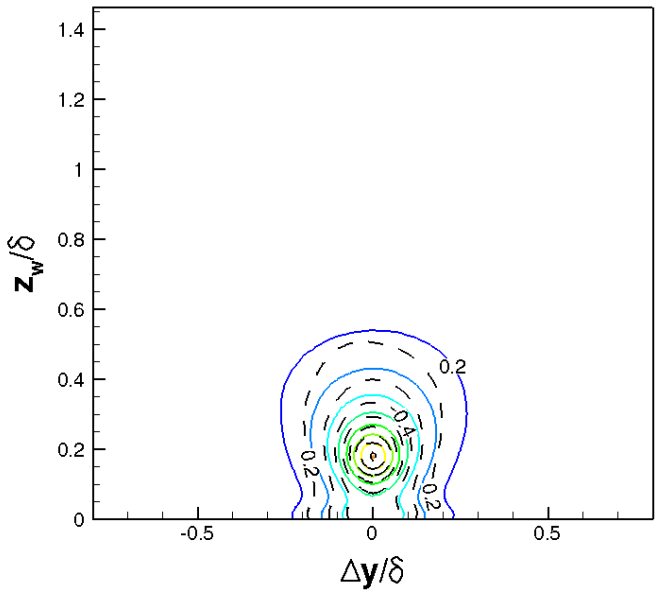

(b) $z_{w, r e f} / \delta=0.18$

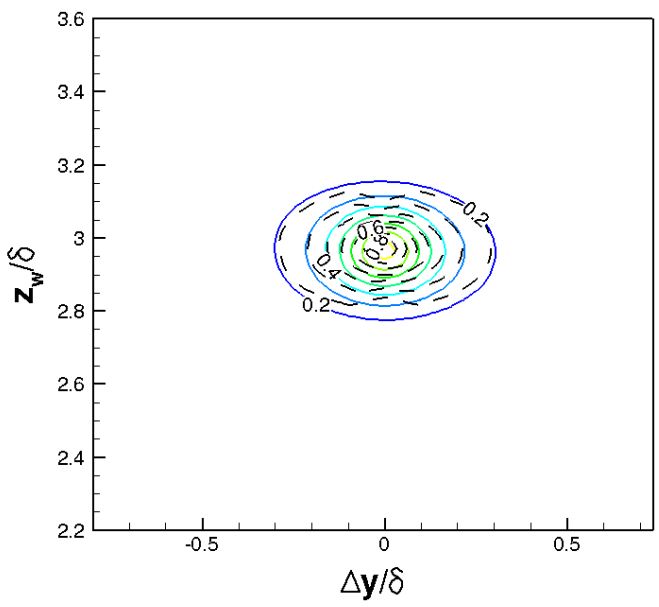

(d) $z_{w, r e f} / \delta=2.97$

Figure 8. Contours of constant azimuthal radial (spanwise wall-normal) correlation coefficient of the pressure signal $C_{p p}(\Delta x, \Delta y)$ at selected heights for Case DNS_HLB (Colored solid line) and Case DNS_FlatPlate (Black dashed line). Case DNS_FlatPlate corresponds to the DNS of hypersonic turbulent boundary layer over a flat plate with $M_{\infty}=5.86$ and $T_{w} / T_{r}=0.76 .^{7}$ Contours $^{7}$ levels vary from 0.2 to 0.9 with increments of 0.1 . 


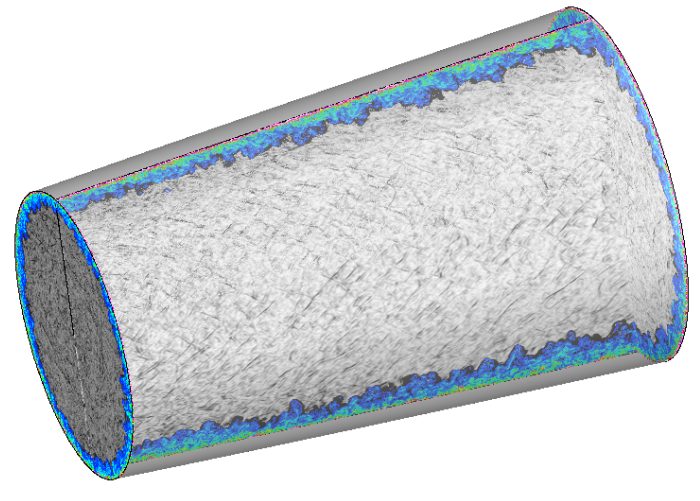

(a)

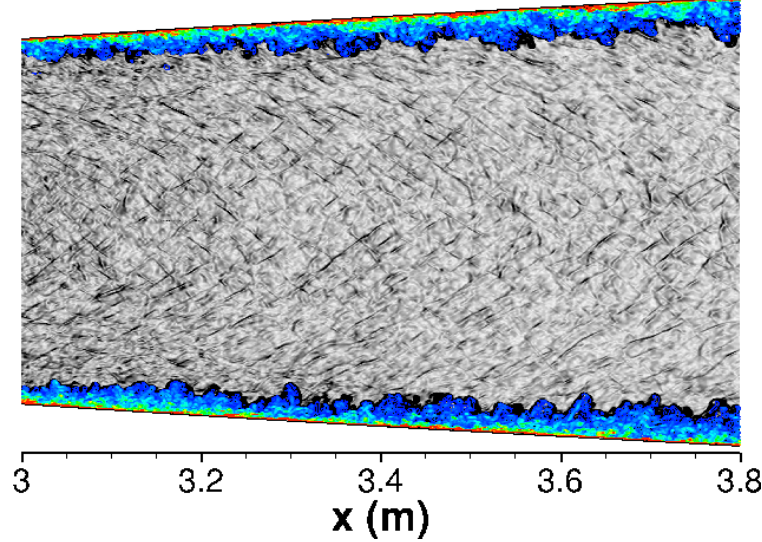

(c)

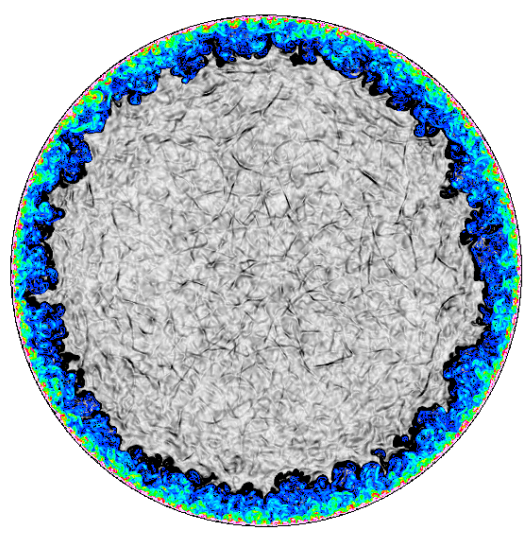

(b)

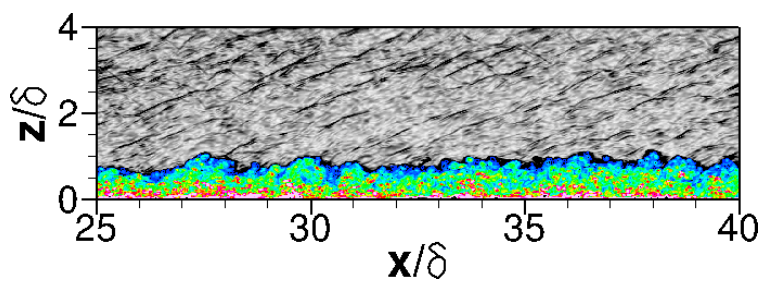

(d)

Figure 9. Numerical schlieren (i.e., density gradients) images of radiation wave of (a) three dimensional volume, $3.0<x<3.8 \mathbf{m}$; (b) cross section at $x=3.7 \mathbf{m}$; (c) streamwise-radial plane $(3.0<x<3.8$ m); (d) streamwise-wall-normal plane of Case DNS_FlatPlate. Case DNS_FlatPlate corresponds to the DNS of hypersonic turbulent boundary layer over a flat plate with $M_{\infty}=5.86$ and $T_{w} / T_{r}=0.76 .^{7}$ Contours are colored by the magnitude of vorticity. 